\title{
Influence of Temperature on the Bolt Loads and Variation in a Bolted Flange for Subsea Pipeline Connection
}

\author{
Pengpeng Wang $(\mathbb{D}$, Lan Zhang $\mathbb{D}$, and Liquan Wang $(\mathbb{D}$ \\ College of Mechanical and Electrical Engineering, Harbin Engineering University, Harbin 150001, Heilongjiang, China \\ Correspondence should be addressed to Lan Zhang; zhanglan@hrbeu.edu.cn
}

Received 23 June 2020; Revised 14 March 2021; Accepted 25 April 2021; Published 5 May 2021

Academic Editor: Weichao Shi

Copyright () 2021 Pengpeng Wang et al. This is an open access article distributed under the Creative Commons Attribution License, which permits unrestricted use, distribution, and reproduction in any medium, provided the original work is properly cited.

\begin{abstract}
This article proposed an analytic and finite element-combined modelling method for the investigation of temperature effects on bolt loads and variation in a bolted flange for subsea pipeline connection. With this method, the preloads of bolt in the bolted flange assembly were investigated under different medium liquid temperatures in the pipeline. The simulation results illustrate that the deviation of bolt loads can be increased due to the medium liquid temperature increasing. The final bolt load deviation increases along with the increasing of the initial deviation of bolt preloads in a constant medium liquid temperature. The final distribution of bolt loads can be affected by the initial condition of the bolts. When bolt preloads are staggered, the variance of the final bolt loads is minimized; when the bolt preloads are axisymmetric, the final bolt load variance is the maximum.
\end{abstract}

\section{Introduction}

Due to the depletion of land oil reserves, more attention is being paid to offshore oil, especially deep-sea resources. As a safe and reliable transportation method, the subsea oil pipeline played a very important role in deep-water oil and gas exploitation. Flange connection is a common way to connect the deep-sea oil pipelines [1-3]. Therefore, it is necessary to study the safety and reliability of flange connection.

The temperature of the pipeline and the flange is not immutable. When the medium liquid goes through the pipeline, the temperature increases, then the bolt loads increase as well. In a bolted flange connection, elastic interaction happens; if a bolt is tightened, it leads to the other bolt loads' increase or decrease. In 1985, Payne found that that the transient temperature change was a major cause of flange connection failure [4]. In 2007, Abid [5, 6] carried out an experiment and showed that when the temperature reached a steady state, the contact stress decreased. In 2010, Omiya and Sawa [7] measured the thermal expansion coefficient and compression resilience of metal wound gaskets and nonasbestos compression flat gaskets under a certain pretightening force. In 2015, Sawa et al. [8] concluded that the pipe flange connection with the spiral wound gasket was expected to keep the superior sealing performance at $300^{\circ} \mathrm{C}$ for a long time. In 2020, Chan et al. [9] proposed a new leakage element that can be widely applied to many vacuum technologies and applications. The studies only took the temperature into account and ignored that the temperature change may influence the distribution of the bolt loads that may lead to the leak of the flange. Since Campen [10] proposed the elastic interaction coefficient method to obtain uniform preload, Takaki and Fukuoka [11], Murali Krishna et al. [12], and Housari et al. [13] all confirmed the correctness of the elastic interaction coefficient method and improved it. However, this method needed to determine the elastic interaction coefficient matrix, which is not an easy job. Previous studies of the sealing performance of flange only focused on either the influence of temperature change or the influence of elastic interaction. In fact, the temperature changes and the elastic interaction should be taken into account at the same time. The fluctuation of the temperature results in the change of the bolt load and then that will cause a further elastic interaction among the bolts. It means that there is a big difference among the bolt loads due to the 
temperature change. With the development of simulation technology, simulation is used in more and more auxiliary works. In 2005, Wang et al. [14] used the three-dimensional finite element method to simulate the distribution of a constant temperature field for the flange joint and the gasket stress, without consideration of the bolt stress. In 2007, Zhou et al. [15] created a three-dimensional mechanical and heat transfer model of bolted flange connection. Considering the nonlinearity and contact of the gasket, the transient temperature distribution of the flange and the influence of temperature on gasket and bolt load were studied, but they only analyzed the change of one bolt load, and the elastic interaction was not taken into account. In 2015, Chen et al. [16] analyzed the temperature distribution at $500^{\circ} \mathrm{C}$ by simulation, and the simulation result was verified experimentally, but they only got the temperature distribution, and the bolt load was not analyzed. In 2020, Začal and Jancar [17] demonstrated the effect of thermal expansion of flange joints and connected pipes on a simplified model by FEM analysis and presented ways of eliminating the negative effects of temperature on the joints based on experimental measurements and practical experience, but the elastic interaction was not taken into account either. Cai and Wang [18] investigated the heat transfer characteristics of a feedthrough flange under a high alternating current excitation by a threedimensional finite element model; the correlations between the heat transfer behaviors and system parameters including applied current, ceramic thickness, and working time were discussed comprehensively; under the natural cooling condition, the correlations among the maximum temperature of the flange, applied current, ceramic thickness, and working time were analyzed systematically, except the gasket stress.

In fact, the distribution of bolt loads is very important for the sealing performance of the flange, which cannot be ignored. This paper proposed an analytic and finite elementcombined modelling method; taking both temperature change and the elastic interaction into account, we obtained the distribution trend of bolt preload with the temperature change with a given initial bolt preload deviation.

\section{The Heat Transfer Model}

As shown in Figure 1, the flange of the subsea pipeline is preloaded by eight bolts (B1-B8), and the medium liquid goes through the pipeline inside.

The heat transfer model includes heat conduction, heat radiation, and heat convection. The calculation of heat radiation is highly nonlinear, including the material nonlinearity and geometric nonlinearity. Heat convection is usually temperature-dependent; it means that the heat convection coefficient cannot be directly calculated, so an appropriate heat transfer model is required. The heat transfer model can be simplified as an equivalent coefficient of thermal conductivity with a good calculation accuracy.

2.1. Equivalent Heat Conductivity Coefficient of the Water Layer between the Bolt and Hole. Although the heat transfer in the seawater layer is low, if it is fully ignored, the temperature difference between the bolt and the flange can be exaggerated. If the seawater layer is treated as a liquid thermal material with a conductivity, a big error can be introduced as well. The heat conduction is the easiest to be calculated on comparing with heat convection and heat radiation, and the coefficient of heat conductivity can be set in the material property in simulation. Therefore, the heat transfer can be treated as an equivalent coefficient of heat conductivity. With the setting of the temperature-dependent equivalent coefficient of heat conductivity, the heat radiation, heat convection, and heat conduction can be simulated. So the heat transfer can be idealized as a heat conduction process with a temperature-dependent equivalent heat conductivity coefficient.

As the real surface is not perfect, the nature of the surface can increase or decrease energy exchange. The direct energy flux between the two surfaces is expressed by an angular factor. The determination of the angle factor is to find the energy exchange between differential area elements of two surfaces and after that integrate it on the whole surface. The result relies on the geometric conditions. The angular factor represents only the percentage of the radiant energy that reaches the other surface, which is independent of the absorption capacity of the other surface. It is a parameter that depends on the size and position of the surfaces only. The heat exchange model between the hole and the bolt can be simplified as a heat exchange case between two concentric cylinders. The two concentric cylinders with the same length, $L$, and the inner surface of the outer cylinder with radius, $r_{2}$, radiate directly to the outer surface of the inner cylinder with radius, $r_{1}$. The angle factor of the hole radiation to the bolt can be obtained [19-21] as follows:

$$
\begin{aligned}
\phi_{1-2}= & R_{1}\left[1-\frac{1}{\pi} \cos ^{-1}\left(\frac{\chi_{1}}{\chi_{2}}\right)\right]+\frac{\sqrt{\chi_{2}^{2}-4 R_{1}^{2}}}{2 \pi R_{2}} \cos ^{-1}\left(R_{1} \frac{\chi_{1}}{\chi_{2}}\right) \\
& +\frac{\chi_{1}}{2 \pi R_{2}} \sin ^{-1} R_{1}-\frac{\chi_{2}}{4 R_{2}}
\end{aligned}
$$

where $\quad R_{1}=r_{1} / r_{2}, \quad R_{2}=L_{1} / r_{2}, \quad \chi_{1}=R_{2}^{2}+R_{1}^{2}-1, \quad$ and $\chi_{2}=R_{2}^{2}+R_{1}^{2}+1$.

Considering the effects of heat radiation, the heat emissivity of the bolt and hole can be expressed as

$$
\varepsilon_{s}=\frac{1}{1+\phi_{1-2}\left(\left(1 / \varepsilon_{1}\right)-1\right)+\phi_{2-1}\left(\left(1 / \varepsilon_{2}\right)-1\right)},
$$

where $\varepsilon_{1}$ and $\varepsilon_{2}$ denote the blackness of the bolt and hole.

As the materials of the bolt and flange are corrosionresistant alloy and heat-resistant alloy, the corrosion of seawater also produces an oxidation layer on the surfaces for the long-term operation in deep water, so the blackness of all parts is selected as 0.8 .

When the heat convection can be ignored, the heat transfers through the heat radiation and heat conduction of the seawater layer; then, the energy that transfers between the flange and the bolt is 

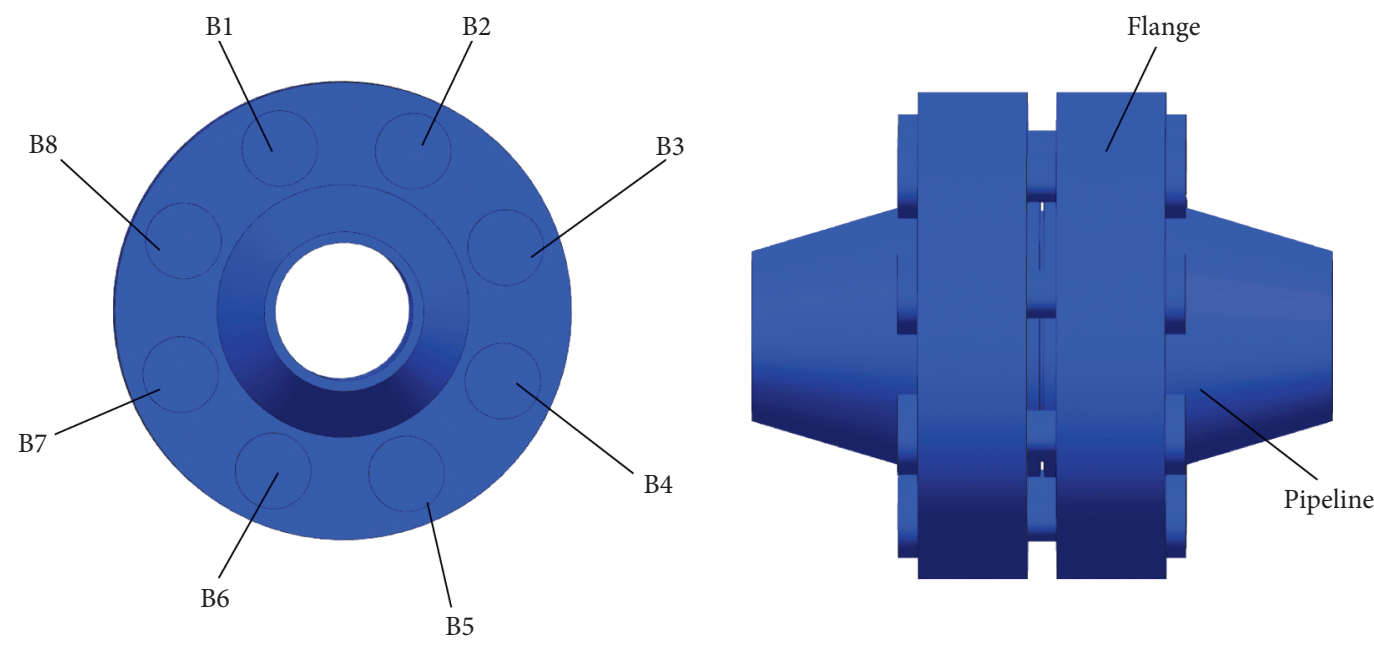

Figure 1: The flange and the bolts.

$$
\begin{aligned}
Q_{1-2} & =2 \pi r_{1} L_{1} \phi_{1-2} \varepsilon_{1} C_{0}\left(\frac{T_{1}}{100}\right)^{4} \\
& =2 \pi L_{1} \lambda_{k 2} \frac{T_{1}-T_{2}}{\operatorname{In}\left(r_{2} / r_{1}\right)}+\varepsilon_{s} 2 \pi r_{1} L_{1} \phi_{1-2} C_{0}\left(\frac{T_{1}^{4}-T_{2}^{4}}{10^{8}}\right),
\end{aligned}
$$

where $Q_{1-2}$ is the heat transfer of the seawater layer between the flange and bolt, $J, T_{1}$ is the bolt surface temperature, $\mathrm{K}, T_{2}$ is the flange bolt hole temperature, $\mathrm{K}, C_{0}$ is the blackbody emissivity, $5.67 \mathrm{~W} /\left(\mathrm{m}^{2} \cdot \mathrm{K}^{4}\right)$, and $\lambda \mathrm{k}^{2}$ is the seawater thermal conductivity at $T_{2}[22], \mathrm{W} /(\mathrm{m} \cdot \mathrm{K})$; the heat transfer can be expressed with the equivalent heat conductivity between the bolt and hole, $\lambda \mathrm{e} 1$ :

$$
Q_{1-2}=2 \pi L_{1} \lambda_{e 1} \frac{T_{1}-T_{2}}{\ln \left(r_{2} / r_{1}\right)}
$$

Substitute equation (4) into (3) and yield

$$
2 \pi r_{1} L_{1} \phi_{1-2} \varepsilon_{1} C_{0}\left(\frac{T_{1}}{100}\right)^{4}=2 \pi L_{1} \lambda_{\mathrm{e} 1} \frac{T_{1}-T_{2}}{\ln \left(r_{2} / r_{1}\right) .}
$$

The equivalent heat conductivity can be expressed as

$$
\lambda_{e 1}=\lambda_{k 2}+r_{1} \varepsilon_{s} \phi_{1-2} C_{0}\left(\frac{T_{1}^{4}-T_{2}^{4}}{10^{8}}\right) \frac{\ln \left(r_{2} / r_{1}\right)}{T_{1}-T_{2}} .
$$

Considering that the heat conductivity is a function of temperature, this equation is a coupling equation, which can be solved by the dichotomy method. The relation of the equivalent heat conductivity coefficient and temperature is plotted in Figure 2.

2.2. Equivalent Heat Conductivity between Flanges. The heat convection between the outer surface of the seal ring and the seawater is much lower than the seawater heat conduction and heat radiation, so it can be ignored in simulation. The heat transfer between the flanges can be treated as the heat conduction and heat radiation, and we yield the following:

$$
\begin{aligned}
2 \pi r l \lambda_{\mathrm{e} 2} \frac{T_{3}-T_{4}}{\ln \left(r_{3} / r_{4}\right)} & =2 \pi r l \lambda_{\mathrm{k} 4} \frac{T_{3}-T_{4}}{\ln \left(r_{3} / r_{4}\right)}+2 \pi r_{1} l C_{0} \varepsilon_{s}\left(\frac{T_{3}^{4}-T_{4}^{4}}{10^{8}}\right), \\
\lambda_{\mathrm{e} 2} & =\lambda_{k 4}+C_{0} \frac{\ln \left(r_{3} / r_{4}\right)}{T_{1}-T_{2}}\left(\frac{T_{3}^{4}-T_{4}^{4}}{10^{8}}\right),
\end{aligned}
$$

where $\lambda_{\mathrm{e} 2}$ is the equivalent heat conductivity between the flanges, $\mathrm{W} /(\mathrm{m} \cdot \mathrm{K}), \lambda_{\mathrm{k} 4}$ is the seawater heat conductivity at $T_{4}$ [22], $\mathrm{W} /(\mathrm{m} \cdot \mathrm{K}), T_{3}$ is the seal surface temperature, $\mathrm{K}, T_{4}$ is the seawater temperature, $\mathrm{K}, r_{3}$ is the seal ring radius, and $r_{4}$ is the flange radius.

The corresponding temperature-dependent equivalent heat conductivity coefficient $\lambda_{\mathrm{e} 2}$ is shown in Figure 3.

\subsection{Equivalent Heat Transfer Coefficient of the Outer Surface} of the Flange. As the flange is exposed to the seawater environment, the main heat transfer is natural convection heat exchange with the seawater, $q_{2}$, and monomer radiation heat transfer, $q_{3}$. The convective heat loss is defined as $Q_{p}$.

The radiative heat exchange between the external surface of the flange and the seawater can be replaced by the equivalent air convection heat exchanger in simulation and calculated as

$$
q_{e}=q_{2}+q_{3}
$$

which is

$$
Q_{p}=h_{p} S_{p}\left(T_{5}-T_{6}\right),
$$

where $Q_{p}$ is the heat loss due to convection heat transfer, J, $h_{p}$ is the seawater natural heat transfer coefficient [23], $200 \mathrm{~W} \cdot \mathrm{m}^{-2} \cdot \mathrm{K}^{-1}, S_{p}$ is the outer surface area of the flange, $\mathrm{m}^{2}$, $T_{5}$ is the flange outer surface temperature, $\mathrm{K}$, and $T_{6}$ is the seawater temperature, $\mathrm{K}$. 


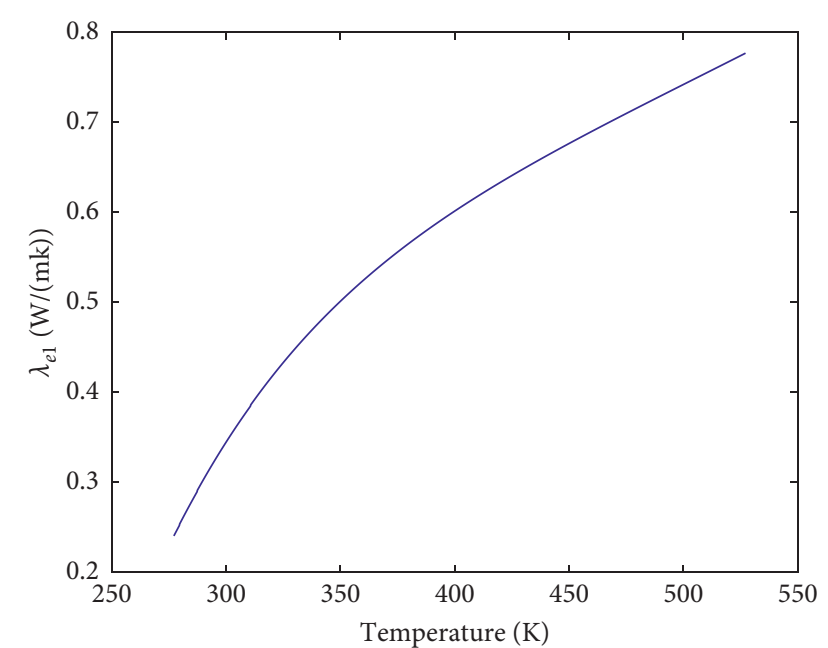

FIGURE 2: The relation of the equivalent heat conductivity coefficient $\lambda_{\mathrm{e} 1}$ and temperature.

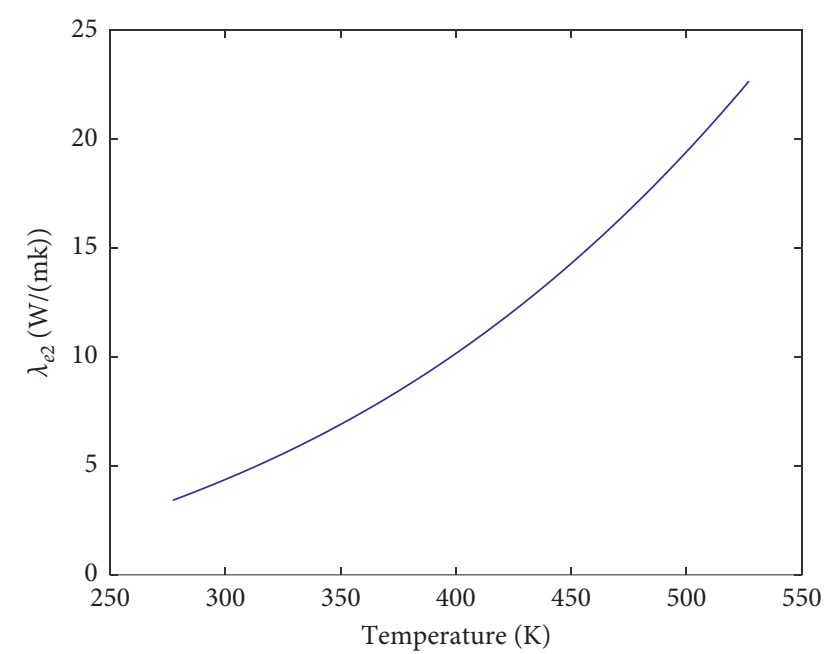

FIGURE 3: The relation of the equivalent heat conductivity coefficient $\lambda_{\mathrm{e} 2}$ and temperature.

In addition, the heat loss due to heat radiation $Q_{R}$ is

$$
Q_{R}=C_{0} \varepsilon S_{p} \frac{T_{5}^{4}-T_{6}^{4}}{10^{8}},
$$

where $Q_{R}$ is the heat loss caused by heat radiation from the outer surface of the pressure ring, J, and $\varepsilon$ is the blackness, 0.8 .

Introducing a temperature-dependent equivalent heat transfer coefficient of convection and heat radiation, $h_{e}$, is

$$
\begin{aligned}
h_{e} S_{p}\left(T_{5}-T_{6}\right) & =h_{p} S_{p}\left(T_{5}-T_{6}\right)+C_{0} \varepsilon S_{p} \frac{T_{5}^{4}-T_{6}^{4}}{10^{8}}, \\
h_{e} & =h_{p}+\frac{C_{0} \varepsilon\left(T_{5}^{4}-T_{6}^{4} / 10^{8}\right)}{T_{5}-T_{6}} .
\end{aligned}
$$

The relation of the equivalent heat transfer coefficient and temperature is plotted in Figure 4.

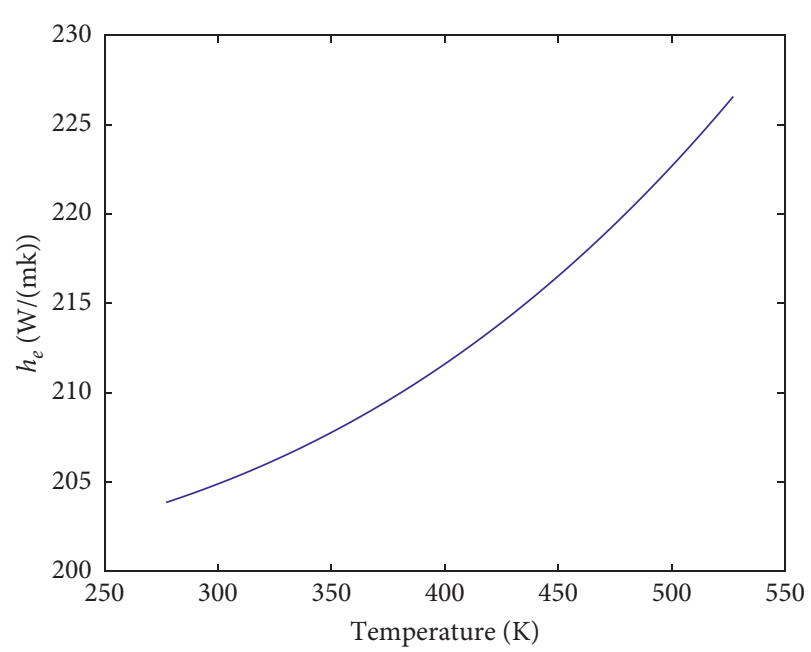

FIgURE 4: The relation of the equivalent heat transfer coefficient $h_{\mathrm{e}}$ and temperature.

\section{Calculation Results and Analysis}

The temperature-dependent conductivity coefficients and heat transfer coefficient acquired in Section 2 can be applied in the flange assembly in the simulation of the thermal process.

3.1. Simulation Settings. The material of the seal ring is $12 \mathrm{Cr} 1 \mathrm{MoV}$, and the material of flange and bolts is $060 \mathrm{~A} 35$ (British Standards Institution). The material properties are listed in Table 1. In the step module, create 2 steps: the first step was static and general to compute the bolt loads, and the second step was coupled temp-displacement to compute the heat transfer process. In the load module, set the boundary conditions: the one flange end was set as fixed, as shown in Figure 5, and the translational degrees of freedom of the $\mathrm{XYZ}$ axis of surface $\mathrm{A}$ is on the flange set as 0 , and the translational degrees of freedom of the XZ axis of surface $\mathrm{B}$ on all the bolts set as 0 , and the translational degree of freedom of the $Y$ axis of surface $\mathrm{C}$ which is on all the bolts were 0 ; then, set the temperature boundary of the inner pipeline as $373 \mathrm{~K}, 423 \mathrm{~K}, 473 \mathrm{~K}$, and $523 \mathrm{~K}$, respectively, in Step 2; in the final, set the bolt loads. In the interaction module, create interaction property Inprop-1, Inprop-2, and Inprop-3 and list in Table 2, and the Inprop-1 was used for the bolts and the hole, the Inprop- 2 was used for the flanges, and the Inprop-3 was used for the outer surface of the flange and the surface of the bolts which contact the water.

3.2. Temperature Field Analysis. The temperature field of the flange is shown in Figure 6. It can be seen that the temperature of the flange gradually decreases from inside to outside, but the temperature of the bolt and hole was not evenly distributed. The temperature at the flange joint decreases significantly from the sealing ring, but the total decrease is lower than that of the flange external surface.

The temperature distribution of the bolt is shown in Figure 7 . The temperature in the middle of the bolt in the 
TABLE 1: Material behaviors.

\begin{tabular}{|c|c|c|c|}
\hline Material & & $12 \mathrm{Cr} 1 \mathrm{MoV}$ & 060A35 \\
\hline \multirow{4}{*}{ Conductivity $\mathrm{W} /(\mathrm{m} \cdot \mathrm{K})$} & $293 \mathrm{~K}$ & 35.6 & \\
\hline & $373 \mathrm{~K}$ & 35.6 & 46.0 \\
\hline & $473 \mathrm{~K}$ & 35.6 & 48.2 \\
\hline & $573 \mathrm{~K}$ & 35.2 & 44.9 \\
\hline Mass density $t / \mathrm{mm}^{3}$ & & $7.85 \times 10^{-9}$ & $7.87 \times 10^{-9}$ \\
\hline \multirow{2}{*}{ Elastic } & Young's modulus $\mathrm{MPa}$ & 210000 & 210000 \\
\hline & Poisson' ratio & 0.3 & 0.3 \\
\hline \multirow{4}{*}{ Expansion coeff. $\mathrm{K}^{-1}$} & $293 \mathrm{~K}$ & $10^{-6}$ & \\
\hline & $373 \mathrm{~K}$ & $1.125 \times 10^{-6}$ & $1.245 \times 10^{-6}$ \\
\hline & $473 \mathrm{~K}$ & $1.218 \times 10^{-6}$ & $1.287 \times 10^{-6}$ \\
\hline & $573 \mathrm{~K}$ & $1.249 \times 10^{-6}$ & $1.350 \times 10^{-6}$ \\
\hline Specific heat $\mathrm{mJ} /(t \cdot \mathrm{K})$ & & $4.6 \times 10^{8}$ & $4.8 \times 10^{8}$ \\
\hline
\end{tabular}

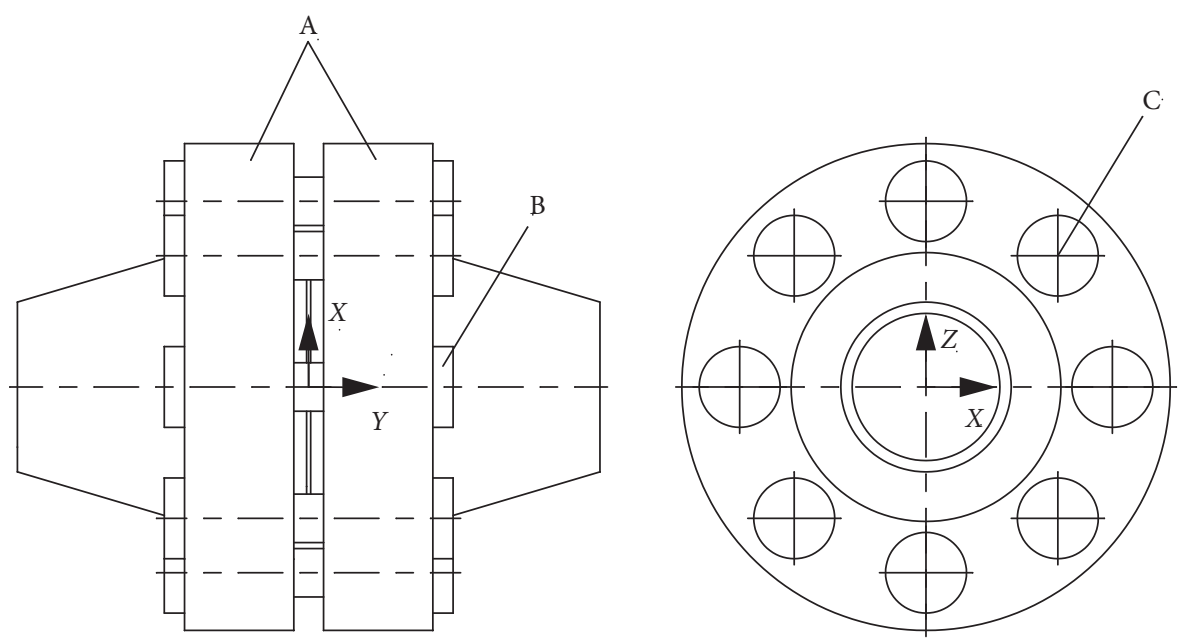

FIgURE 5: The boundary conditions.

TABLE 2: Inprops in the interaction property.

\begin{tabular}{|c|c|c|c|c|c|c|c|c|}
\hline \multirow{3}{*}{ Type } & \multicolumn{3}{|c|}{ Inprop-1 } & \multicolumn{3}{|c|}{ Inprop-2 } & \multicolumn{2}{|c|}{ Inprop-3 } \\
\hline & \multicolumn{3}{|c|}{ Contact } & \multicolumn{3}{|c|}{ Contact } & \multicolumn{2}{|c|}{ Film conduction } \\
\hline & $\begin{array}{l}\text { Conductance } \\
\mathrm{W} \cdot \mathrm{m}^{-2} \cdot \mathrm{K}^{-1}\end{array}$ & $\begin{array}{c}\text { Clearance } \\
\mathrm{mm}\end{array}$ & $\begin{array}{c}\text { Temp. } \\
\text { K }\end{array}$ & $\begin{array}{l}\text { Conductance } \\
\mathrm{W} \cdot \mathrm{m}^{-2} \cdot \mathrm{K}^{-1}\end{array}$ & $\begin{array}{c}\text { Clearance } \\
\mathrm{mm}\end{array}$ & $\begin{array}{c}\text { Temp. } \\
\text { K }\end{array}$ & $\begin{array}{l}\text { Film coeff. } \\
\mathrm{W} \cdot \mathrm{m}^{-2} \cdot \mathrm{K}^{-1}\end{array}$ & Temp. K \\
\hline & 0.241 & 3 & 273 & 3.421 & 5 & 273 & 203.861 & 273 \\
\hline & 0.353 & 3 & 298 & 4.474 & 5 & 298 & 205.003 & 298 \\
\hline & 0.440 & 3 & 323 & 5.670 & 5 & 323 & 206.350 & 323 \\
\hline & 0.506 & 3 & 348 & 7.030 & 5 & 348 & 207.922 & 348 \\
\hline & 0.560 & 3 & 373 & 8.574 & 5 & 373 & 209.731 & 373 \\
\hline & 0.605 & 3 & 398 & 10.321 & 5 & 398 & 211.798 & 398 \\
\hline & 0.644 & 3 & 423 & 12.287 & 5 & 423 & 214.138 & 423 \\
\hline & 0.679 & 3 & 448 & 14.489 & 5 & 448 & 216.797 & 448 \\
\hline & 0.713 & 3 & 473 & 16.942 & 5 & 473 & 219.709 & 473 \\
\hline & 0.745 & 3 & 498 & 19.660 & 5 & 498 & 222.971 & 498 \\
\hline & 0.777 & 3 & 523 & 22.662 & 5 & 523 & 226.578 & 523 \\
\hline
\end{tabular}

flange hole is the highest and gradually decreases to both ends. The distributions of the temperature from the inside to the outside along the axial direction of the bolt are similar, but the outside is slightly different due to the influence of heat dissipation from the two end faces of the bolt, and the radial direction is nonlinear and gradually decreases due to the influence of the seawater layer.
3.3. The Change of Bolt Loads. Because of the elastic interaction, the bolt preloads are not exactly the same. If the temperature of the flange changes, the bolt loads change as well. When the bolt loads change, the elastic interaction between the bolts further affects the uniformity of the bolt loads which affects the sealing of the flange in the final [24]. Therefore, the temperature change affects the sealing of the 


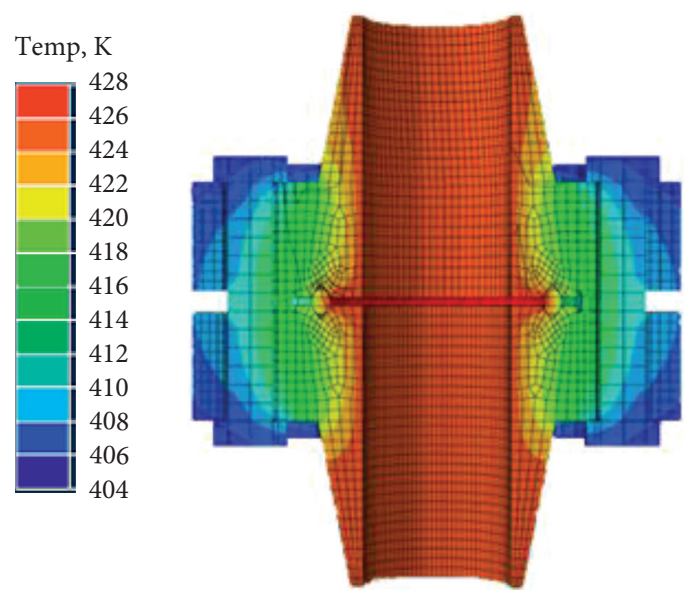

FIgURE 6: Flange temperature distribution.

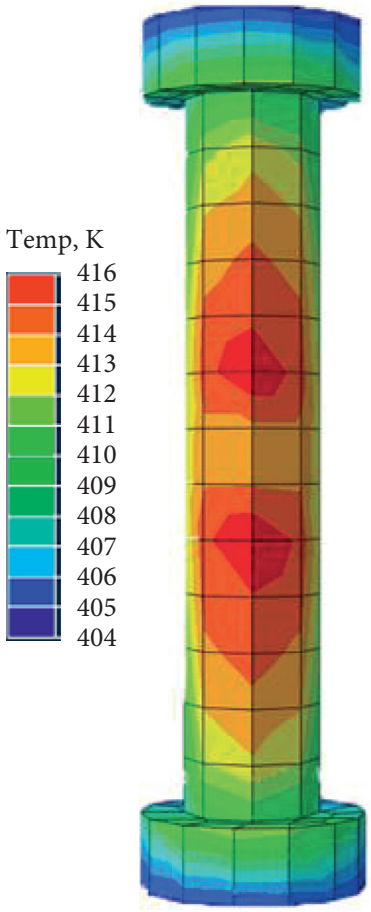

FIgURE 7: Bolt temperature distribution.

flange as a result. In order to study the influence of the temperature change on the uniformity of bolt preloads' distribution, this study investigates the bolt load change under different temperatures with the proposed finite element method.

The simulation shows that when the bolts' preload is uniform and the medium liquid temperature is $373 \mathrm{~K}$ (Condition 1), the bolt loads change are shown in Figure 8.

When the bolt preloads are uniform, the medium liquid temperature is $473 \mathrm{~K}$ (Condition 2), and the bolt loads' change is shown in Figure 9.

When the bolts' preload is uniform, the medium liquid temperature is $523 \mathrm{~K}$ (Condition 3), and the bolt loads' change is shown in Figure 10.

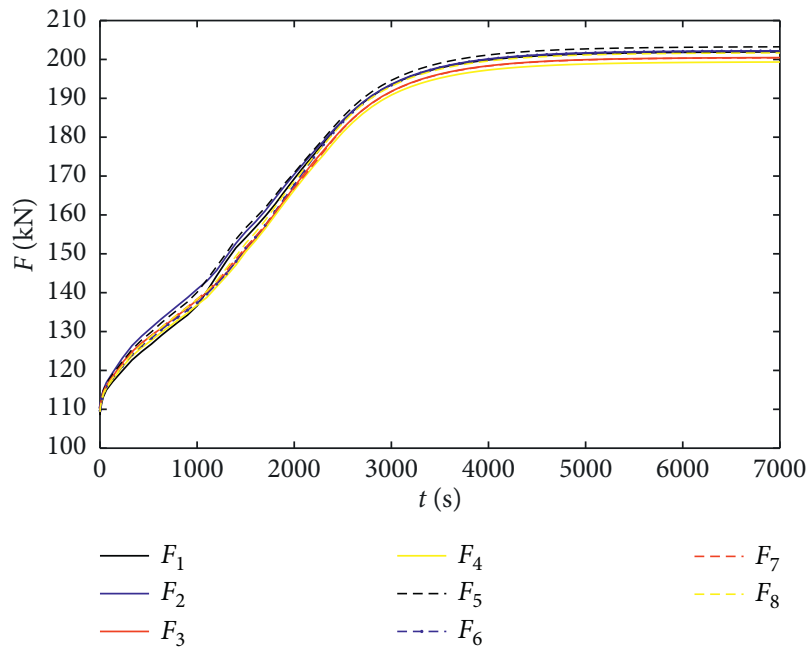

Figure 8: The bolt loads change with time in Condition 1 (373 K).

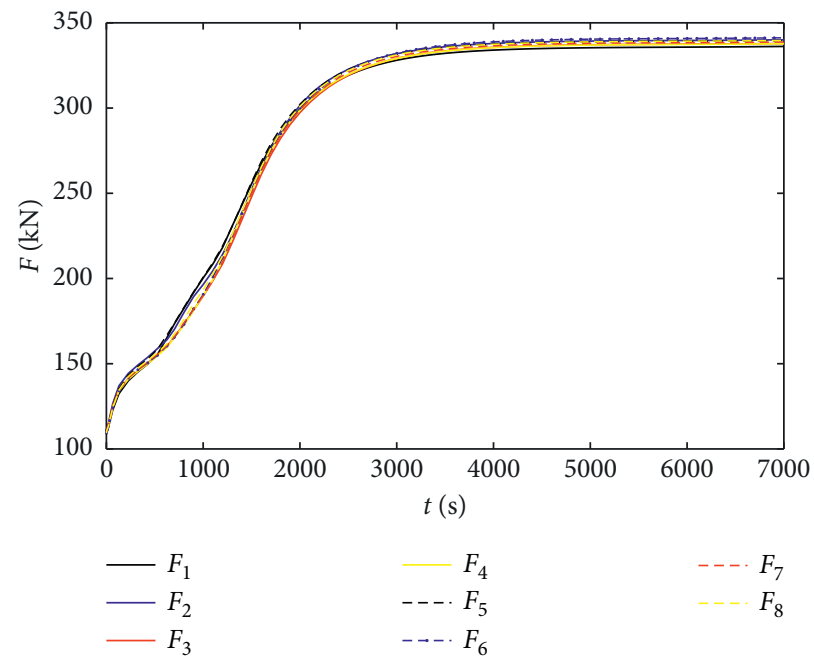

FIgURE 9: The bolt loads change with time in Condition 2 (473 K). 


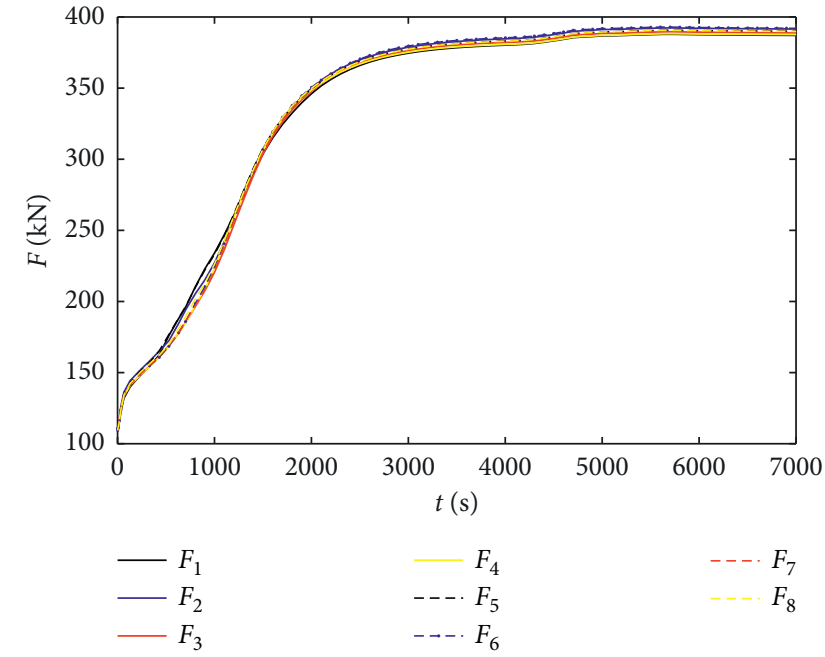

Figure 10: The bolt loads change with time in Condition 3 (523 K).

When the bolts' preload variation is $1 \%$ and the temperature of the medium liquid is $523 \mathrm{~K}$ (Condition 4), the bolt loads' change is shown in Figure 11.

When the bolts' preload variation is $2 \%$ and the temperature of the medium liquid is $523 \mathrm{~K}$ (Condition 5), the bolt loads' change is shown in Figure 12.

When the bolts' preload variation is $5 \%$ and the temperature of the medium liquid is $523 \mathrm{~K}$ (Condition 6), the bolt loads' change is shown in Figure 13.

The bolt loads increase quickly from 0 to $2000 \mathrm{~s}$ and after that gently until stable.

Then, we can see that the high temperature of the medium liquid leads to a high increase of the bolt load, and the bolt loads are more scattered; meanwhile, a big bolt preload deviation leads to more scattered bolt loads; in order to study the effects of the distribution of the bolts' preload on the bolt loads with the same deviation, define the standard deviation as

$$
S=\sqrt{\frac{\sum\left(F_{i}-\mu_{F}\right)^{2}}{8}}
$$

where $S$ is the standard deviation, $F_{i}$ is the bolt load of bolt $i$, $i=1-8$, and $\mu_{F}$ is the average of the bolt loads.

When the bolt preload deviation is $5 \%$, the temperature of the medium liquid is $523 \mathrm{~K}$, and the bolts' preload was axial symmetry (Condition 7), the standard deviation is shown in Figure 14.

When the bolt preload deviation is $5 \%$, the temperature of the medium liquid is $523 \mathrm{~K}$, and the bolts' preload was decreasing (Condition 8), the standard deviation is shown in Figure 15.

When the bolt preload deviation is $5 \%$, the temperature of the medium liquid is $523 \mathrm{~K}$, and the bolt preloads are staggered (Condition 9), the standard deviation is shown in Figure 16.

The bolt load peak deviations are around $6-6.4 \mathrm{kN}$, happen around $1000 \mathrm{~s}$, and tend to be stable after $2000 \mathrm{~s}$.

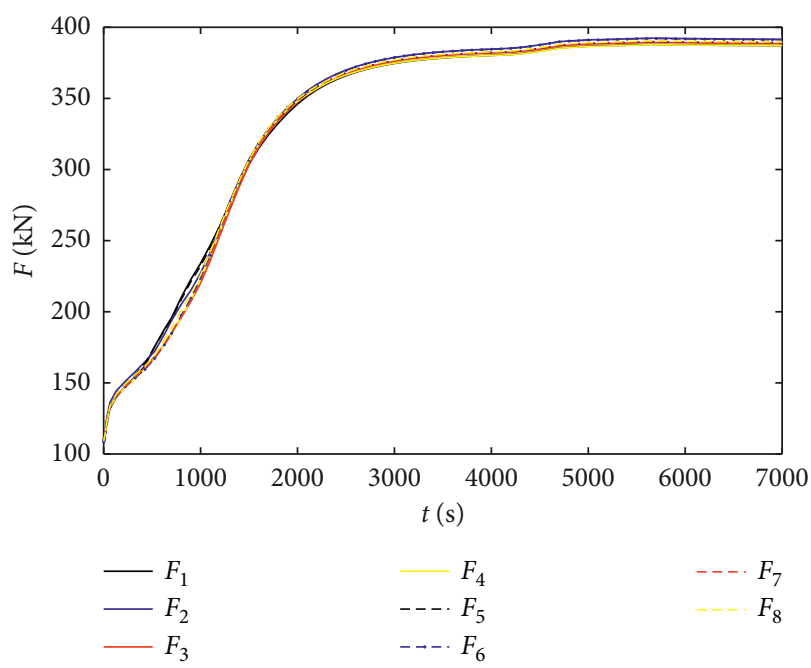

Figure 11: The bolt loads change with time in Condition 4.

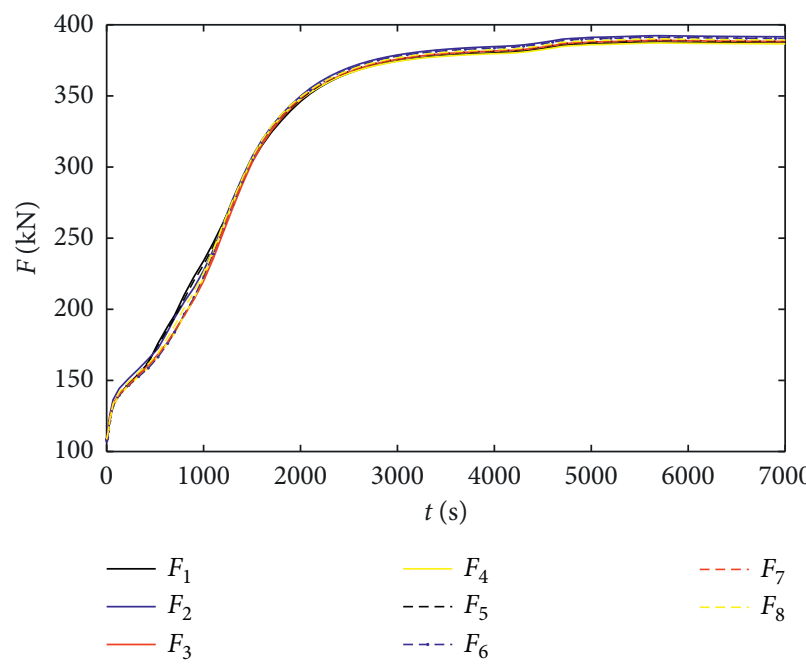

FIgUre 12: The bolt loads change with time in Condition 5.

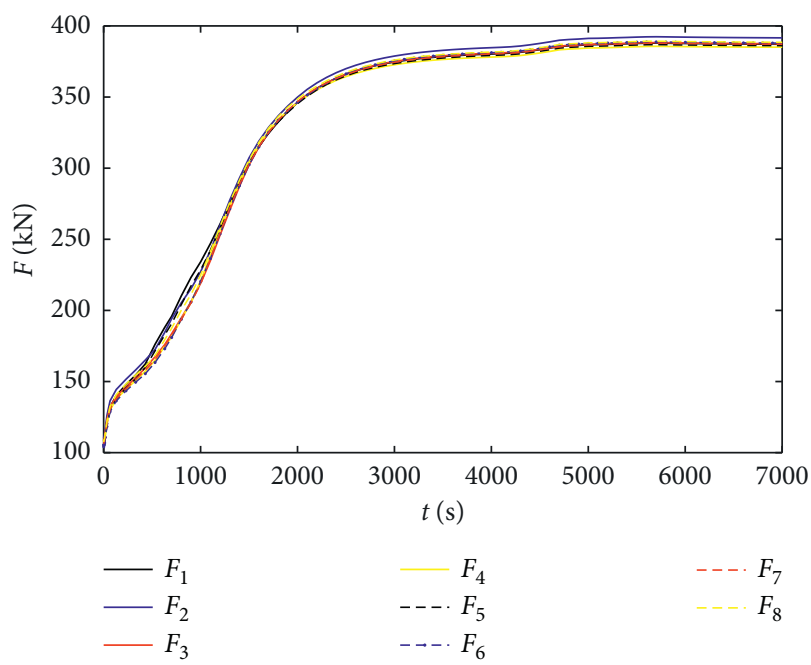

FIgURE 13: The bolt loads change with time in situation 6 . 


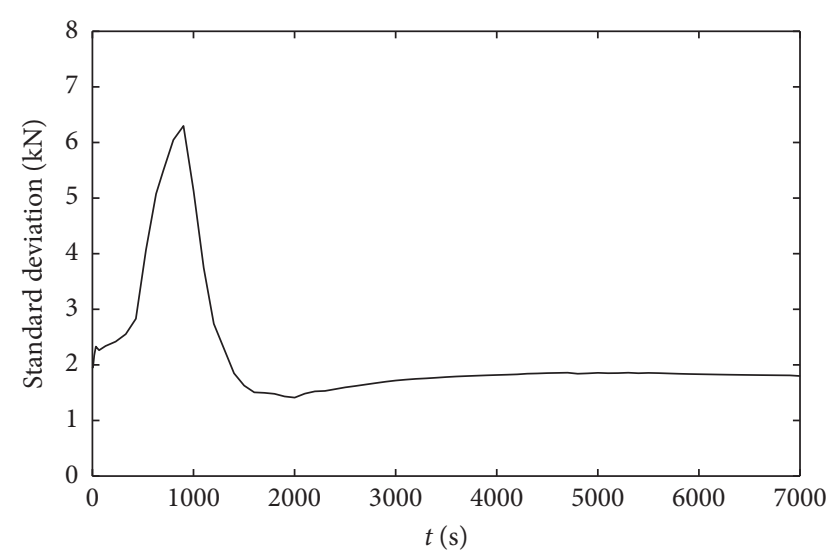

Figure 14: The standard deviation with time in Condition 7.

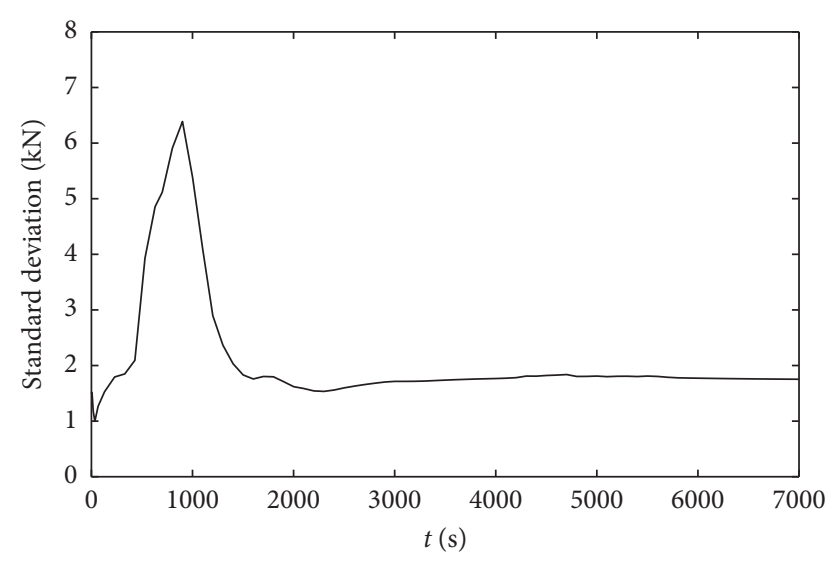

FIgure 15: The standard deviation with time in Condition 8.

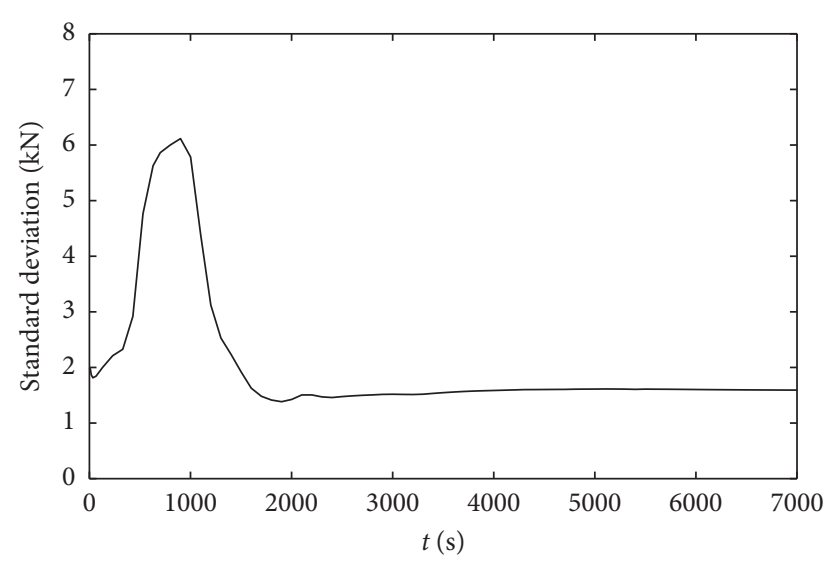

FIgure 16: The standard deviation with time in Condition 9.

\section{Conclusion and Discussion}

This research proposed an analytic and finite elementcombined modelling approach to analyze the thermalstructural coupling for bolted flanges of pipeline connection. With the proposed method, a bolted flange was analyzed and the following conclusions can be drawn:
(1) As the temperature of the liquid in the pipe increased, the temperature of the flanges and bolts increased as well, resulting in the bolt load increase. At the same time, as the temperature increased, the bolt loads' standard deviation increased first and then decreased; the maximum standard deviation of the final load was $1.800 \mathrm{kN}$; the maximum standard deviation in the thermal process was $6.388 \mathrm{kN}$.

(2) The temperature of the medium liquid also influenced the distribution of the bolt loads; a higher temperature of the medium liquid led to a more uneven final load distribution.

(3) If the deviation of the bolt preloads was a constant, the distribution of bolts' preload also affected the final load distribution of the bolts. When the bolts' preload was staggered, the variance of the final bolt loads was minimized; when the bolt preloads decreased, the final bolt load variance increased, and the maximum standard deviation was about $6.388 \mathrm{kN}$.

\section{Data Availability}

The data used to support the findings of this study are included within the article.

\section{Conflicts of Interest}

The authors declare that they have no conflicts of interest.

\section{Acknowledgments}

This work was supported by the National Natural Science Foundation of China, Grant no. 51779064.

\section{References}

[1] Z. G. Li, Q. H. Lin, D. Y. Zhao, and L. Q. Wang, "The structural design and optimization for the inner-frame of subsea flange connection tool," Key Engineering Materials, vol. 419-420, pp. 197-200, 2009.

[2] G. Corbetta and R. Cruden, "A new approach to capex and opex reduction: an integrated system for remote tie-ins and pipeline repair," in Proceedings of the Offshore Technology Conference, pp. 313-319, Houston, TX, USA, May 2000.

[3] N. Bouchonneau, V. Sauvant-Moynot, D. Choqueuse et al., "Experimental testing and modelling of an industrial insulated pipeline for deep sea application," Journal of Petroleum Science and Engineering, vol. 73, pp. 111-112, 2010.

[4] J. R. Payne, "PVRC flanged joint user's survey," Welding Research Council, vol. 306, 1985.

[5] M. Abid, "Determination of safe operating conditions for gasketed flange joint under combined internal pressure and temperature: a finite element approach," International Journal of Pressure Vessels and Piping, vol. 83, no. 6, pp. 433-441, 2006.

[6] M. Abid, J. A. Chattha, and K. A. Khan, "Finite element analysis of a gasketed flange joint under combined internal pressure and thermal transient loading," in Proceedings of the ASME Pressure Vessels and Piping Conference, pp. 261-267, San Antonio, TX, USA, July 2007. 
[7] Y. Omiya and T. Sawa, "Asme, Thermal stress analysis and the sealing performance evaluation of bolted flange connection at elevated temperature," in Proceedings of the ASME Pressure Vessels and Piping Conference, pp. 397-408, Prague, Czech Republic, July 2009.

[8] T. Sawa, Y. Takagi, and H. Torii, "Effect of material properties of gasket on the sealing performance of pipe flange connections at elevated temperature," in Proceedings of the ASME 2015 Pressure Vessels and Piping Conference, Boston, MA, USA, July 2015.

[9] C. K. Chan, S. D. Yeh, C. C. Chang et al., "A flange-type standard leak element and its vacuum applications," Vacuum, vol. 184, Article ID 109945, 2021.

[10] D. H. Van Campen, Systematic Bolt-Tighting Procedure for Reactor Vessle Flanges, pp. 10-15, University of Technology, The Delft, Netherlands, 1969.

[11] T. Takaki and T. Fukuoka, "Effective bolting up procedure using finite element analysis and elastic interaction coefficient method," in Proceedings of the ASME/JSME 2004 Pressure Vessels and Piping Conference, San Diego, CA, USA, July 2004.

[12] M. Murali Krishna, M. S. Shunmugam, and N. Siva Prasad, "A study on the sealing performance of bolted flange joints with gaskets using finite element analysis," International Journal of Pressure Vessels and Piping, vol. 84, no. 6, 2007.

[13] B. A. Housari, A. A. Alkelani, and S. A. Nassar, "A proposed model for predicting clamp load loss due to gasket creep relaxation in bolted joints," Journal of Pressure Vessel Technology, vol. 134, no. 2, Article ID 021201, 2012.

[14] J. H. Wang, R. L. Cai, and W. M. Zhong, "3 -D finite element analysis on flanged joints under static temperature field," Pressure Vessel Technology, vol. 10, pp. 21-25, 2005.

[15] X. J. Zhou, X. Q. Qiu, B. Zhang, and G. M. Chen, "Threedimensional finite element analysis of bolted flange joint with transient temperature," Lubrication Engineering, vol. 32, no. 6, pp. 64-67, 2007.

[16] Y. Chen, K. S. Guan, K. Ma, L. Z. Zhang, and Y. Y. Xu, "Analysis on temperature distribution and heat loss of bolted flanged connections at high temperature," Journal of East China University of Science and Technology, vol. 6, pp. 857862, 2015.

[17] J. Začal and L. Jancar, "Effect of temperature on bolt working load of pressure vessels," in Current Methods of Construction DesignSpringer, Berlin, Germany, 2020.

[18] X. Cai and H. Wang, "Heat transfer analysis of feedthrough flange under high alternating current condition," Science China Technological Sciences, vol. 63, no. 4, pp. 686-692, 2020.

[19] J. A. Stevenson and J. C. Grafton, Radiation Heat Transfer Analysis for Space Vehicles, pp. 61-119, Aeronautical Systems Division Air Force Systems Command United States Air Force Wright-patterson Air Force Base, Columbus, OH, USA, 1961.

[20] X. M. Zhang and Z. P. Ren, Heat transfer, China Building Industry Press, Beijing, China, 2001.

[21] X. J. Zhou, Research on Transient Sealing Design Method of Large Caliber Flange at High Temperature, China University of Petroleum, Beijing, China, 2008.

[22] M. H. Sharqawy, "New correlations for seawater and pure water thermal conductivity at different temperatures and salinities," Desalination, vol. 313, no. 11, pp. 97-104, 2013.

[23] Y. Takeuchi, T. Guo, and A. Li, Thermal stress, pp. 49-51, Science Press, Beijing, China, 1977.

[24] T. Sawa and H. Shimazu, "FEM stress analysis and the sealing performance evaluation in stainless steel fittings under internal pressure," Case where Internal Fluid Is Liquid, vol. 1, pp. 275-276, 2004. 